\title{
Evaluation of Gallium Arsenide Thermal Expansion Coefficient by Extended X-Ray Absorption Fine Structure
}

\author{
Gora Dieye' ${ }^{1}$, Sameh I. Ahmed ${ }^{2}$, Abdou C. Wade1, Djibril Diop ${ }^{1}$ \\ ${ }^{1}$ Département de Physique, Faculté des Sciences et Techniques, Université Cheikh Anta Diop, Dakar, Senegal \\ ${ }^{2}$ Department of Physics, Faculty of Science, Ain Shams University, Cairo, Egypt \\ Email: dieyegora@gmail.com, abouwade@yahoo.com,d_diop@yahoo.com,sameh2967@gmail.com
}

How to cite this paper: Dieye, G., Ahmed, S.I., Wade, A.C. and Diop, D. (2019) Evaluation of Gallium Arsenide Thermal Expansion Coefficient by Extended X-Ray Absorption Fine Structure. World Journal of Condensed Matter Physics, 9, 37-46.

https://doi.org/10.4236/wjcmp.2019.92003

Received: February 1, 2019

Accepted: March 10, 2019

Published: March 13, 2019

Copyright $\odot 2019$ by author(s) and Scientific Research Publishing Inc. This work is licensed under the Creative Commons Attribution International License (CC BY 4.0).

http://creativecommons.org/licenses/by/4.0/

\begin{abstract}
Negative thermal expansion of gallium arsenide has been investigated through temperature dependent Extended X-ray Absorption Fine Structure (EXAFS) measurements. The bond thermal expansion coefficient $\alpha_{\text {bond }}$ has been evaluated and compared to negative expansion coefficient $\alpha_{\text {tens }}$ due to tension effects. The overall thermal expansion coefficient is the sum of $\alpha_{\text {bond }}$ and $\alpha_{\text {tens }}$. Below $60 \mathrm{~K}, \alpha_{\text {tens }}$ is greater than $\alpha_{\text {bond }}$ yielding to a negative expansion in this temperature region. Tension effects are progressively overcome by the stretching effects in the region $60-300 \mathrm{~K}$. The asymmetry of nearest neighbors distribution is not negligible since the gaussian approximation underestimates the bond expansion by about $0.00426 \AA$. This error decreases when the temperature is lowered. The accuracy in the thermal expansion evaluation and the connection between third cumulant and thermal expansion are discussed.
\end{abstract}

\section{Keywords}

Negative Thermal Expansion, Tension Effects, EXAFS, Asymmetry, Gallium Arsenide

\section{Introduction}

Thermal expansion is a physical parameter defined as "the tendency of matter to change in shape, area, and volume in response to change in temperature" [1]. It is a key parameter in many scientific and technological applications [2] [3] [4] [5].

EXAFS (Extended X-ray Absorption Fine Structure) is a powerful tool for 
studying the local thermal expansion of crystals [6] [7]. Anharmonicity effects of the effective pair potential on EXAFS have been revealed by studies on several systems [8] [9] [10], even at low temperatures, showing that the standard harmonic treatment of disorder in EXAFS [11] was inadequate. The cumulant approach [12] [13] is particularly suitable for treating moderately disordered systems. Specific information can be obtained by considering the values of the cumulants and their variation with temperature [14] or pressure [15].

As a correlation sensitive technique, EXAFS probes the unidimensional distribution of instantaneous distances $r=\left|\boldsymbol{r}_{b}-\boldsymbol{r}_{a}\right|$. This sensitivity of EXAFS to local structure of crystals is shared by other techniques such as total scattering [16]. The bond thermal expansion measured by EXAFS is given by the temperature dependence of the average value $\langle r\rangle$. Contrary to EXAFS, diffraction technique measures the crystallographic distance $R_{c}=\left|\left\langle\boldsymbol{r}_{b}\right\rangle-\left\langle\boldsymbol{r}_{a}\right\rangle\right|$. The thermal expansion measured by EXAFS is always larger than the crystallographic one measured by Bragg diffraction due to perpendicular vibrations which increase with temperature [14]. By comparing bond lengths measured by EXAFS and Bragg diffraction, one can evaluate the perpendicular Mean Square Relative Displacement (MSRD) which is related to the vibrations normal to the bond. The EXAFS technique has the great advantage to disentangle parallel and perpendicular vibrations contributions on thermal expansion [17].

For a two-atomic system, the thermal expansion is the result of the interaction potential anharmonicity and is always positive, but this explanation cannot be extended to crystals [18] where thermal expansion is the sum of a positive contribution due to bond-stretching effects and a negative one due to tension effects [19]. These central force mechanisms were detailed by Bruno et al. [20]. Negative thermal expansion (NTE) occurs only when tension effects prevail over bond stretching contribution.

In some framework structures such as $\mathrm{ZrW}_{2} \mathrm{O}_{8}$ where NTE is observed in large temperature interval [21], tension effects are often related to low-frequency rigid unit modes (RUMs) [22]. There are however other framework structures like $\mathrm{CuScO}_{2}$ [23] and $\mathrm{Ag}_{2} \mathrm{O}$ [24] where NTE is not attributed to RUMs. Moreover, simpler structures like tetrahedral semiconductors also exhibit a weak NTE in limited temperature range [25] [26].

In EXAFS studies, the average values of the bond thermal expansion coefficient were generally obtained by linearly fitting the temperature dependence of the bond length [17] [24] [27]. The main drawback of this method is that the non-linear low temperature behaviour of thermal expansion coefficient is not evidenced [28]. We propose in this work a more refined evaluation of the temperature dependence of the bond expansion. It is based on an Einstein fit to temperature bond length variation and had never been used to evaluate thermal expansion coefficient of GaAs.

The purpose of this study, based on high quality EXAFS data at both Ga and As $\mathrm{K}$ edges, was to determine the temperature dependence of thermal expansion 
coefficient $\alpha(T)$ in order to obtain a deeper understanding of correlation between lattice negative expansion and anharmonicity.

Section 2 describes the experimental procedure. In Section 3, after a comparison between crystallographic and bond expansions, we evaluate the coefficient of thermal expansion. Section 4 is dedicated to a discussion on the effect of distribution asymmetry in the thermal expansion evaluation. The effect of asymmetry is further explored in Section 5 through the relation between third cumulant and thermal expansion.

\section{Experiment}

EXAFS spectra of Ga and As $\mathrm{K}$ edges were recorded in transmission mode at the XAFS beamline of Elettra with an electron energy of $2 \mathrm{GeV}$ and current of 300 $\mathrm{mA}$. The used monochromator consists of two silicon crystals with parallel reflecting faces (111). A reflection from a Pt-coated mirror was used to reduce the relative influence of harmonics.

$0.02 \mathrm{~g}$ of GaAs was mixed with $0.20 \mathrm{~g}$ of graphite fine powder to obtain samples in form of pellets. The sample homogeneity was checked by scanning the sample using narrow vertical and horizontal collimating slits and by analyzing the transmitted $\mathrm{x}$-rays distribution on a phosphorus screen behind the sample. Measurements were done such that the X-ray beam impinges on the largest homogeneous region of the sample. Two ionization chambers filled with krypton gas at pressures 140 and 500 mbar were used to measure the incoming and outgoing photon fluxes, respectively. Another pellet of GaAs was inserted before a third ionization chamber and served as reference for energy calibration. The sample was mounted on a liquid-He cryostat, on which a thermocouple was fixed to vary temperatures. The temperature was varied in the interval $14-350$ $\mathrm{K}$, at 25 or $50 \mathrm{~K}$ steps. Three spectra were recorded at each temperature for each edge.

The edge jump $\Delta \mu x$ was about 1.06 at the $\mathrm{Ga} \mathrm{K}$ edge $(10,367 \mathrm{eV})$ and 0.97 at the As $\mathrm{K}$ edge $(11,867 \mathrm{eV})$. The energy of the incident X-rays was scanned in the ranges $E=10,130-11,745 \mathrm{eV}$ (for Ga) and $E=11,627-13,393 \mathrm{eV}$ (for As), with a $\Delta E$ step varying from $0.2 \mathrm{eV}$ in the near-edge region to $5 \mathrm{eV}$ at the end of the spectra, in order to obtain a uniform wavevector step $\Delta k=0.025 \AA$ in the EXAFS region.

The data analysis was carried out using the standard procedure and already detailed in [29] [30].

\section{Expansion Coefficients}

For moderate vibrational disorder, the EXAFS signal can be expanded in terms of the cumulants of the unidimensional distribution of distances $\rho(r)$. The first cumulant $C_{1}^{*}=\langle r\rangle$ corresponds to the mean value, the second cumulant $C_{2}^{*}=\left\langle(r-\langle r\rangle)^{2}\right\rangle$ is the mean square displacement and the third cumulant $C_{3}^{*}=\left\langle(r-\langle r\rangle)^{3}\right\rangle$ represents the mean cubic displacement. Equation (1) shows 
the relation between the crystallographic distance $R_{c}$ and the mean distance [31]

$$
\langle r\rangle \simeq R_{c}+\frac{\left\langle\Delta u_{\perp}^{2}\right\rangle}{2 R_{c}}
$$

$\left\langle\Delta u_{\perp}^{2}\right\rangle$ is the perpendicular mean square relative displacement (MSRD).

The Ga-As bond expansion $\delta\langle r\rangle$ obtained with EXAFS cumulant analysis and the crystallographic expansion $\delta R_{c}$ are compared in Figure 1. $R_{c}$ values were quoted from Novikova [32] (stars items in the figure), Leszczynsky [33] (cross items) and Smith and White [25] (diamond items).

The bond expansion is positive in the full temperature range and, as expected, is always greater than the crystallographic expansion due to perpendicular vibrations to the bond. The crystallographic expansion is negative up to $60 \mathrm{~K}$ where contribution from tension effects prevails over bond stretching contribution.

The discrepancy between the results of Ga and As edges can be attributed to a leakage of Ga EXAFS on the As EXAFS [29] since the distance between the two edges is approximately $19.3 \AA^{-1}$ in k-space.

Absolute values of $\left\langle\Delta u_{\perp}^{2}\right\rangle$ were evaluated by inverting Equation (1) and fitting a correlated Einstein model to experimental values $2 R_{c}\left[R_{c}-\langle r\rangle\right]$ [17]. The values $-\frac{\left\langle\Delta u_{\perp}^{2}\right\rangle}{2 R_{c}}$ account for negative contribution to crystallographic expansion due to tension effects.

The coefficient of bond expansion is defined as

$$
\alpha_{\text {bond }}(T)=\frac{1}{\langle r\rangle}\left(\frac{\partial\langle r\rangle}{\partial T}\right)_{P}
$$

The same definition holds for $\alpha_{\text {tens }}(T)$ where tension effects contribution is differentiated with respect to $T$. Traditionally, average values were obtained by linearly fitting bond expansion measured by EXAFS or total scattering but this approach had the drawback to miss the non linear behaviour at low temperatures.

To evaluate the expansion coefficients $\alpha_{\text {bond }}(T)$ and $\alpha_{\text {tens }}(T)$ we need to first approximate experimental points. Here we chose an Einstein-like function $y=A+B \operatorname{coth}(C / T)$ where $\mathrm{A}, \mathrm{B}$ and $\mathrm{C}$ are free parameters [34]. The next step is then to derive with respect to temperature the two best-fitting Einstein-like curves to obtain $\alpha_{\text {bond }}(T)$ and $\alpha_{\text {tens }}(T)$.

Both NTE and positive bond expansion increase with temperature and NTE is greater up to about $60 \mathrm{~K}$. This behaviour corresponds to the domination of $-\alpha_{\text {tens }}(T)$ over $\alpha_{\text {bond }}(T)$ as depicted by Figure 2 . In $\mathrm{Cu}$ where negative thermal expansion is not reported, $\alpha_{\text {bond }}(T)$ prevails over $-\alpha_{\text {tens }}(T)$ in the full studied temperature range [35]. For tetrahedral crystals, the strength and temperature interval of NTE increase with ionicity [36] with $\mathrm{CuCl}$ having the maximum values [30]. 


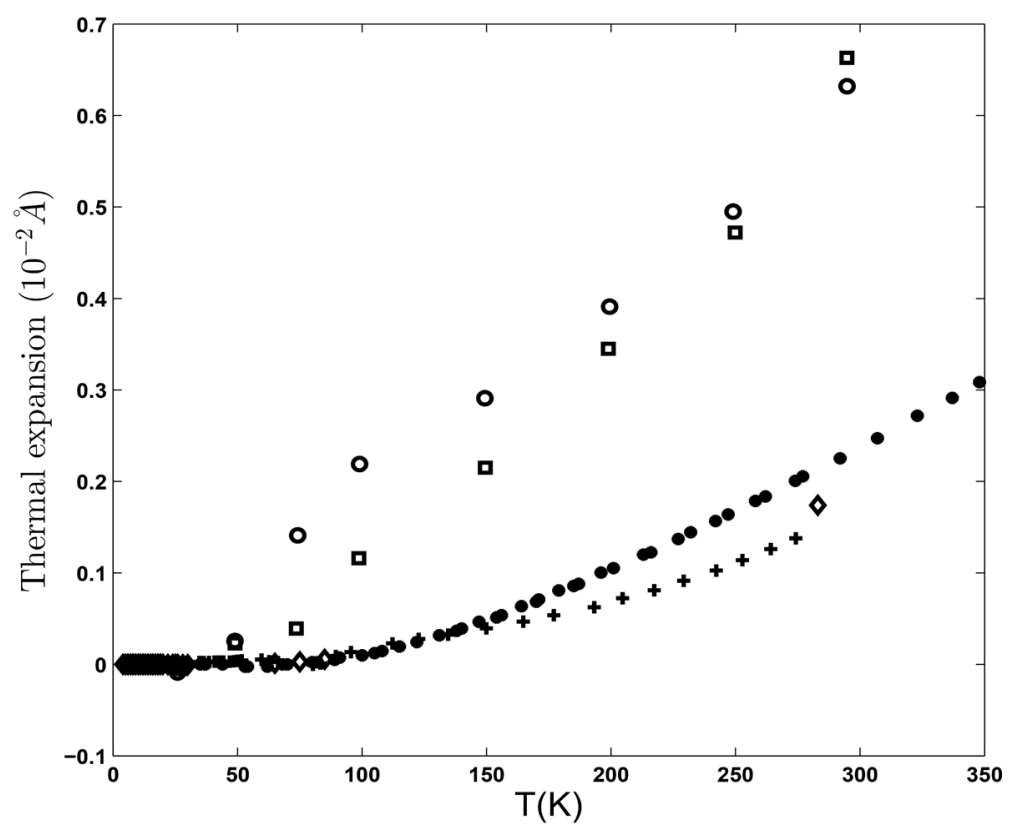

Figure 1. Ga-As bond expansion (circles and squares for As and Ga edges, respectively) and crystallographic expansions from different authors.

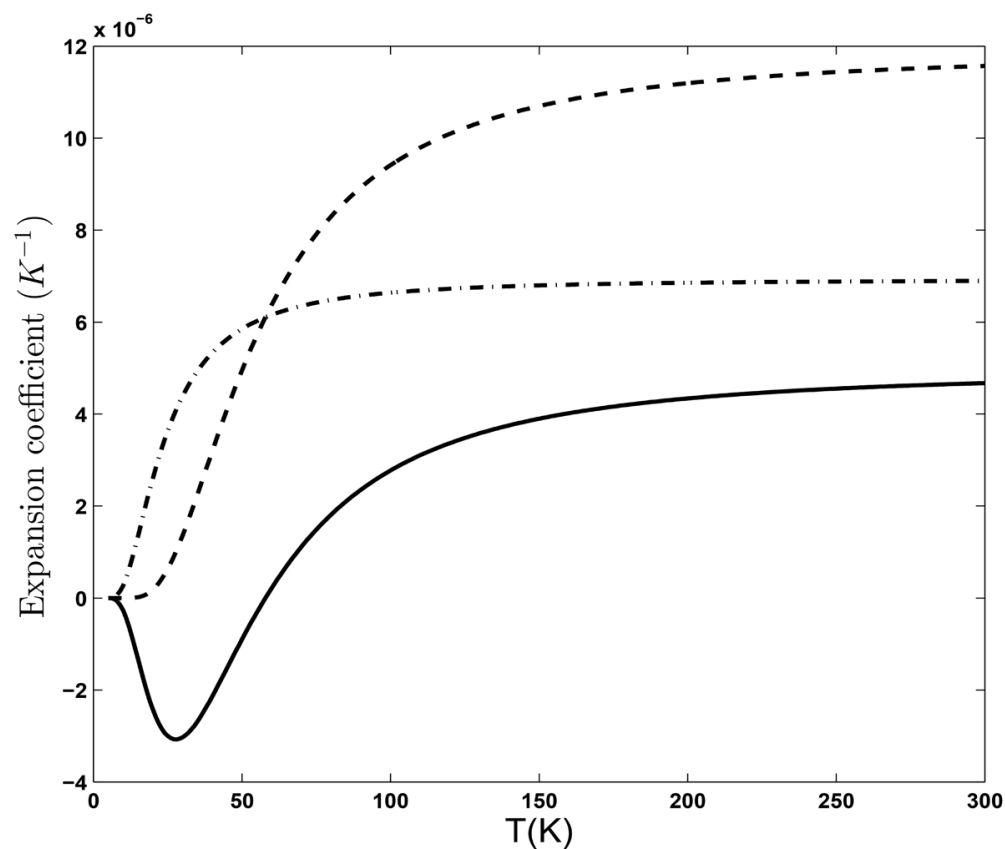

Figure 2. Coefficient of positive expansion $\alpha_{b o n d}(T)$ (dashed line) and negative expansion $\alpha_{\text {tens }}(T)$ (dash-dotted line) $\left(-\alpha_{\text {tens }}(T)\right.$ is plotted for convenience). The continuous line represent the sum $\alpha_{\text {bond }}(T)+\alpha_{\text {tens }}(T)$.

\section{Distribution Asymmetry}

The third cumulant $C_{3}^{*}$ measures the asymmetry of distances distribution $\rho(r)$ and is important in the bond distance and expansions evaluation procedure. The data analysis will lead to more accurate values of bond distances [37] if 
the third cumulant is taken into account.

The phase difference between 14 and $300 \mathrm{~K}$ is shown in Figure 3 for different independent measurements. It is fitted with the thin horizontal line when the asymmetry is neglected. In this case, the variations of first cumulant are 0.00018 $\AA$ and $0.00236 \AA$ for the effective and real distributions, respectively. Now when we take into account the asymmetry, the phase difference is fitted by the oblique line. Here, the first cumulant variations for effective and real distributions are $0.00426 \AA$ and $0.00662 \AA$, respectively.

The real bond expansion of GaAs is thus underestimated by about $0.00426 \AA$ with the Gaussian approximation.

A common observation shared by GaAs [29] with some other structures (diamond-zincblende [17], cuprite [24]) is that strengths of NTE and positive bond expansion have the same variation. This correlation would be underestimated if the distribution asymmetry were neglected.

\section{Third Cumulant and Thermal Expansion}

Thermal expansion can be alternatively measured through the third cumulant [6] [7].

In the case of two-atomic systems, the net expansion is given, through a perturbative quantum approach, to first order by equation [38]

$$
a(T) \simeq-\frac{3 k_{3}}{k_{0}} C_{2}^{*}(T)
$$

where $k_{3}$ can be experimentally obtained from the temperature dependence of $C_{3}^{*}$. The expansion in Equation (3) is solely due to the anharmonicity of the effective pair potential [31].

The situation is different for crystals where the effective pair potential is the result of the statistically averaged behavior of all the atoms in the crystal and can then be temperature dependent [39]. Thus, the bond thermal expansion can also depend on the shift of the minimum of the effective pair potential with respect to the distance axis [35]. This observation was confirmed in copper by path-integral Monte Carlo [40] and in germanium by Molecular Dynamics simulations [41].

So, the thermal expansion (first cumulant) is the sum of two contributions: asymmetry (third cumulant) and shift of the effective potential. The latter is mainly due to perpendicular vibrations to the bonds [31].

In Figure 4, the bond thermal expansion $\delta C_{1}^{*}$ is compared to the thermal expansion due only to asymmetry $\delta a$ and the crystallographic expansion $\delta R_{c}$. As generally expected in crystals, $\delta C_{1}^{*}$ is different from $\delta a$. The latter is larger than $\delta R_{c}$.

Though any direct information on thermal expansion can be obtained from third cumulant, his inclusion in the data analysis is important to obtain accurate values of the bond expansion, especially for the first shell [37]. 


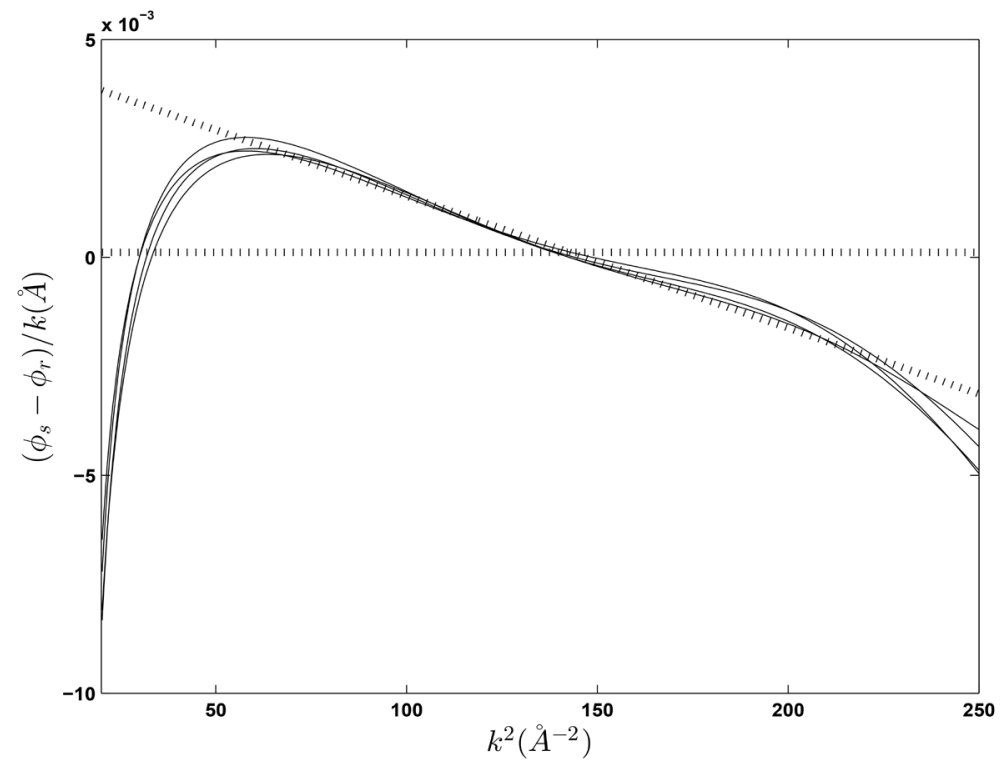

Figure 3. Phase difference $(\phi(300)-\phi(14)) / k$ versus $k^{2}$ at Ga edge for different independent measurements.

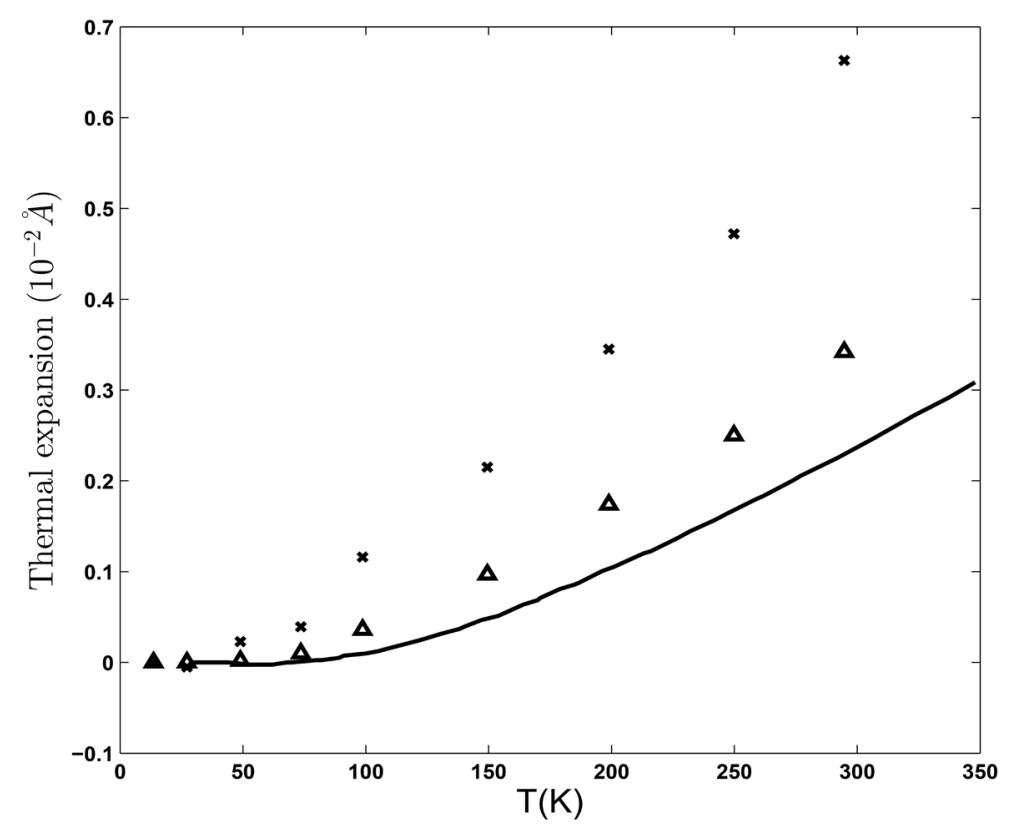

Figure 4. Comparison between thermal expansion measured by EXAFS first cumulant and the contribution due to the asymmetry of distribution for GaAs. The cross represent $\delta C_{1}^{*}$ and triangles $\delta a$. The continuous line is $\delta R_{c}$.

\section{Conclusion}

The coefficient of bond thermal expansion $\alpha_{\text {bond }}(T)$ has been calculated from temperature dependent EXAFS measurements on GaAs. By comparing EXAFS and crystallographic expansions, a coefficient of negative expansion has been evaluated. The overall thermal expansion can be positive or negative depending on whether $\alpha_{b o n d}(T)$ or $\alpha_{\text {tens }}(T)$ prevails in the considered temperature 
range. In order to get accurate values of bond expansion, distribution asymmetry must be taken into account. The present results will be used later to calculate mode Grüneisen parameters. This will help to clarify the connection between the local dynamical behaviour and the average thermodynamical properties of matter.

\section{Acknowledgements}

The authors are grateful to Paolo Fornasini of University of Trento for helpful discussions.

\section{Conflicts of Interest}

The authors declare no conflicts of interest regarding the publication of this paper.

\section{References}

[1] Tipler, P.A. and Mosca, G. (2008) Physics for Scientists and Engineers. Worth Publishers, New York.

[2] Evans, J.S.O. (1999) Negative Thermal Expansion Materials. Journal of Chemical Society, Dalton Transactions, 19, 3317-3326. https://doi.org/10.1039/a904297k

[3] Roy, R., Agarwal, D.K. and McKinstry, H.A. (1989) Very Low Thermal Expansion Coefficient Materials. Annual Review of Material Science, 19, 59-81. https://doi.org/10.1146/annurev.ms.19.080189.000423

[4] Lind, C. (2012) Two Decades of Negative Thermal Expansion Research: Where Do We Stand? Materials, 5, 1125-1154. https://doi.org/10.3390/ma5061125

[5] Takenaka, K. (2012) Negative Thermal Expansion Materials: Technological Key for Control of Thermal Expansion. Science and Technology of Advanced Materials, 13, Article ID: 013001. https://doi.org/10.1088/1468-6996/13/1/013001

[6] Tröger, L., Yokoyama, T., Arvanitis, D., Lederer, T., Tischer, M. and Baberschke, K. (1993) Determination of Bond Lengths, Atomic Mean-Square Relative Displacements, and Local Thermal Expansion by Means of Soft X-Ray Photoabsorption. Physical Review B, 49, 888-903. https://doi.org/10.1103/PhysRevB.49.888

[7] Dalba, G., Fornasini, P., Grisenti, R. and Purans, J. (1999) Anharmonicity and Thermal Expansion in Crystalline Germanium. Journal of Synchrotron Radiation, 6, 253-254. https://doi.org/10.1107/S0909049598017105

[8] Tranquada, J.M. and Ingalls, R. (1983) Extended X-Ray-Absorption Fine Structure Study of Anharmonicity in CuBr. Physical Review B, 28, 3520-3528. https://doi.org/10.1103/PhysRevB.28.3520

[9] Dalba, G., Fornasini, P., Gotter, R. and Rocca, F. (1995) Anharmonicity Effects on the Extended X-Ray-Absorption Fine Structure: The Case of $\beta$ AgI. Physical Review B, 52, 149-157. https://doi.org/10.1103/PhysRevB.52.149

[10] Dalba, G., Diop, D., Fornasini, P. and Rocca, F. (1994) An EXAFS Study of Thermal Disorder in GaAs. Journal of Physics. Condensed Matter, 6, 3599. https://doi.org/10.1088/0953-8984/6/19/016

[11] Lee, P.A., Citrin, P.H., Eisenberger, P. and Kincaid, B.M. (1981) Extended X-Ray Absorption Fine Structure-Its Strengths and Limitations as a Structural Tool. Reviews of Modern Physics, 53, 769-806. https://doi.org/10.1103/RevModPhys.53.769 
[12] Bunker, G. (1983) Application of the Ratio Method of EXAFS Analysis to Disordered Systems. Nuclear Instruments and Methods in Physics Research, 207, 437-444. https://doi.org/10.1016/0167-5087(83)90655-5

[13] Crozier, E.D., Rehr, J.J. and Ingalls, R. (1995) X-Ray Absorption. John Wiley and Sons, New York.

[14] Dalba, G., Fornasini, P., Grisenti, R. and Purans, J. (1999) Sensitivity of Extended X-Ray-Absorption Fine Structure to Thermal Expansion. Physical Review Letter, 82, 4240-4243. https://doi.org/10.1103/PhysRevLett.82.4240

[15] Tranquada, J.M. and Ingalls, R. (1986) X-Ray-Absorption Study of CuBr at High Pressure. Physical Review B, 34, 4267. https://doi.org/10.1103/PhysRevB.34.4267

[16] Egami, T. and Billinge, S.J.L. (2003) Underneath the Bragg Peaks: Structural Analysis of Complex Materials.

[17] Abd el All, N., Dalba, G., Diop, D., Fornasini, P., Grisenti, R., Mathon, O., Rocca, F., Thiodjio Sendja, B. and Vaccari, M. (2012) Negative Thermal Expansion in Crystals with the Zincblende Structure: An EXAFS Study of CdTe. Journal of Physics: Condensed Matter, 24, Article ID: 115403. https://doi.org/10.1088/0953-8984/24/11/115403

[18] Aschroft, N.W. and Mermin, N.D. (1976) Solid State Physics. Thomson Brooks/Cole.

[19] Barrera, G.D., Bruno, J.A.O., Barron, T.H.K. and Allan, N.L. (2005) Negative Thermal Expansion. Journal of Physics: Condensed Matter, 17, R217. https://doi.org/10.1088/0953-8984/17/4/R03

[20] Bruno, J.A.O., Allan, N.L., Barron, T.H.K. and Turner, A.D. (1998) Thermal Expansion of Polymers: Mechanisms in Orthorhombic Polyethylene. Physical Review $B$, 58, 8416-8427. https://doi.org/10.1103/PhysRevB.58.8416

[21] Mary, T.A., Evans, J.S.O., Vogt, T. and Sleight, A.W. (1996) Negative Thermal Expansion from 0.3 to 1050 Kelvin in $\mathrm{ZrW}_{2} \mathrm{O}_{8}$. Science, 272, 90-92. https://doi.org/10.1126/science.272.5258.90

[22] Heine, V., Welche, P.R.L. and Dove, M.T. (1999) Geometrical Origin and Theory of Negative Thermal Expansion in Framework Structures. Journal of the American Ceramic Society, 82, 1793-1802. https://doi.org/10.1111/j.1151-2916.1999.tb02001.x

[23] Tao, J.Z. and Sleight, A.W. (2003) The Role of Rigid Unit Modes in Negative Thermal Expansion. Journal of Solid State Chemistry, 173, 442-448. https://doi.org/10.1016/S0022-4596(03)00140-3

[24] Sanson, A., Rocca, F., Dalba, G., Fornasini, P., Grisenti, R., Dappiagi, M. and Artioli, G. (2006) Negative Thermal Expansion and Local Dynamics in $\mathrm{Cu}_{2} \mathrm{O}$ and $\mathrm{Ag}_{2} \mathrm{O}$. Physical Review B, 73, Article ID: 214305. https://doi.org/10.1103/PhysRevB.73.214305

[25] Smith, T.F. and White, G.K. (1975) The Low-Temperature Thermal Expansion and Grüneisen Parameter of Some Tetrahedrally Bonded Solids. Journal of Physics C: Solid State Physics, 8, 2031-2042. https://doi.org/10.1088/0022-3719/8/13/012

[26] White, G.K. (2006) Solids: Thermal Expansion and Contraction. Contemporary Physics, 34, 193-204. https://doi.org/10.1080/00107519308213818

[27] Ahmed, S.I., Dalba, G., Fornasini, P., Vaccari, M., Rocca, F., Sanson, A., Li, J. and Sleight, A.W. (2009) Negative Thermal Expansion in Crystals with the Delafossite Structure: An Extended X-Ray Absorption Fine Structure Study of $\mathrm{CuScO}_{2}$ and $\mathrm{CuLaO}_{2}$. Physical Review B, 79, Article ID: 104302.

https://doi.org/10.1103/PhysRevB.79.104302 
[28] Touloukian, Y.S., Kirby, R.K., Taylor, R.E. and Desay, P.D. (2008) Thermophysical Properties of Matter. Vol. 13, Plenum, New York.

[29] Ahmed, S.I., Aquilanti, G., Novello, N., Olivi, L., Grisenti, R. and Fornasini, P. (2013) Local Vibrational Properties of GaAs Studied by Extended X-Ray Absorption Fine Structure. The Journal of Chemical Physics, 139, Article ID: 164512. https://doi.org/10.1063/1.4826629

[30] Vaccari, M., Grisenti, R., Fornasini, P., Rocca, F. and Sanson, A. (2007) Negative Thermal Expansion in $\mathrm{CuCl}$ : An Extended X-Ray Absorption Fine Structure Study. Physical Review B, 75, Article ID: 184307. https://doi.org/10.1103/PhysRevB.75.184307

[31] Fornasini, P., Monti, F. and Sanson, A. (2001) Anharmonicity in EXAFS of Crystals. Journal of Synchrotron Radiation, 8, 1214-1220. https://doi.org/10.1107/S0909049501014923

[32] Novikova, S.I. (1961) Fiz. Tverd. Tela 3, 178. [English Transl.: Soviet. Phys. Solid State, 3, 129].

[33] Leszczynski, M., Pluzhnikov, V.B., Czopnik, A., Bak-Misiuk, J. and Slupinski, T. (1997) Thermal Expansion of GaAs: Te and AlGaAs: Te at Low Temperatures. Journal of Applied Physics, 82, 4678-4680. https://doi.org/10.1063/1.366207

[34] Fornasini, P. and Grisenti, R. (2014) The Coefficient of Bond Thermal Expansion Measured by Extended X-Ray Absorption Fine Structure. The Journal of Chemical Physics, 141, Article ID: 164503. https://doi.org/10.1063/1.4899073

[35] Fornasini, P., Beccara, S., Dalba, G., Grisenti, R., Sanson, A., Vaccari, M. and Rocca, F. (2004) Extended X-Ray Absorption Fine Structure Measurements of Copper: Local Dynamics, Anharmonicity and Thermal Expansion. Physical Review B, 70, Article ID: 174301. https://doi.org/10.1103/PhysRevB.70.174301

[36] Fornasini, P., Grisenti, R., Dappiagi, M., Agostini, G. and Miyanaga, T. (2017) Nearest-Neighbour Distribution of Distances in Crystals from Extended X-Ray Absorption Fine Structure. The Journal of Chemical Physics, 147, Article ID: 044503. https://doi.org/10.1063/1.4995435

[37] Sanson, A. (2009) On the Neglecting of Higher-Order Cumulants in EXAFS Data Analysis. Journal of Synchrotron Radiation, 16, 864-868. https://doi.org/10.1107/S0909049509037716

[38] Frenkel, A.I. and Rehr, J.J. (1993) Thermal Expansion and X-Ray Absorption Fine Structure Cumulants. Physical Review B, 48, 585-588. https://doi.org/10.1103/PhysRevB.48.585

[39] Mustre de Leon, J., Conradson, S.D., Batistić, I., Bishop, A.R., Raistrick, I.D., Aronson, M.C. and Garzon, F.H. (1992) Axial Oxygen-Centered Lattice Instabilities in $\mathrm{YBa}_{2} \mathrm{Cu}_{3} \mathrm{O}_{7}$ : An Application of the Analysis of Extended X-Ray-Absorption Fine Structure in Anharmonic Systems. Physical Review B, 45, 2447-2457.

https://doi.org/10.1103/PhysRevB.45.2447

[40] Beccara, S.A. and Fornasini, P. (2008) Path-Integral Monte Carlo Calculation of the Effects of Thermal Disorder in Extended X-Ray-Absorption Fine Structure of Copper. Physical Review B, 77, Article ID: 172304. https://doi.org/10.1103/PhysRevB.77.172304

[41] Sanson, A. (2010) Local Dynamical Properties of Crystalline Germanium and Their Effects in Extended X-Ray Absorption Fine Structure. Physical Review B, 81, Article ID: 012304. https://doi.org/10.1103/PhysRevB.81.012304 